\title{
COMMUTATION PROPERTIES OF THE COEFFICIENT MATRIX IN THE DIFFERENTIAL EQUATION OF AN INNER FUNCTION
}

\author{
STEPHEN L. CAMPBELL
}

\begin{abstract}
Let $A(x)$ be an operator valued function that is analytic on the real axis. Assume that $A(x)$ is selfadjoint for each real $x$. It is shown that $A(x)$ and $\int_{0}^{x} A(s) d s$ commute for all real $x$ iff $A(x)$ and $A(y)$ commute for all real $x$ and $y$. This result is then used to establish several new characterizations of the Potapov inner functions of normal operators $T$ such that $\|T\|<1$. The case where $\|T\|_{1}=1, r(T)<1$ and $A_{T}(x)$ and $A_{T}(y)$ commute for real $x$ and $y$ is discussed. Here $A_{T}(x)=-i U_{T}^{\prime}(x) U_{T}(x)^{*}$ and $U_{T}(x)$ is the Potapov inner function for $T$.
\end{abstract}

This paper is a continuation of the author s study of differentiable inner functions begun in [1], [2], and [4]. It answers some of the questions raised in [1] and improves the characterization of $A_{T}$ for normal $T$ given in [4]. The results of [4] on the $L^{1}$ norm of $A_{T}$ have recently been improved and elaborated on by Helson [7] and Herrero [9].

1. Main result. The main result of this paper is the following theorem. It answers the major unresolved question left in [1]. By operator we mean a bounded linear operator acting in a fixed separable Hilbert space $h$. If $X$ and $Y$ are operators, then $[X, Y]=X Y-Y X$.

THEOREM 1. Suppose that $A(x)$ is an operator valued function which is analytic on the real axis. Suppose further that $A(x)$ is selfadjoint for every real $x$. Then the following are equivalent.

(*) $[A(x), A(y)]=0$ for all real $x$ and $y$.

(**) $\left[A(x), \int_{0}^{x} A(y) d y\right]=0$ for all real $x$.

To prove this result we need the following lemma.

Received by the editors November 3, 1972.

AMS (MOS) subject classifications (1970). Primary 47A45; Secondary 34A30.

Key words and phrases. Operator valued inner function, Potapov inner function, exponential representation, normal operators, commutation properties.

(c) American Mathematical Society 1974 
LEMMA 1. If $T_{1}, T_{2}$ are selfadjoint operators and $\left[T_{1},\left[T_{1}, T_{2}\right]\right]=0$, then $\left[T_{1}, T_{2}\right]=0$.

Proof. Suppose $T_{1}$ and $T_{2}$ are selfadjoint and that $\left[T_{1},\left[T_{1}, T_{2}\right]\right]=0$. Then $\left[T_{1}, T_{2}\right]$ is quasinilpotent [11, p. 4]. But $i\left[T_{1}, T_{2}\right]$ is selfadjoint since $T_{1}$ and $T_{2}$ are. Thus $i\left[T_{1}, T_{2}\right]=0$ since it is a selfadjoint quasinilpotent operator.

Theorem 1 is written in the notation of [1]. For purposes of a proof, it is notationally more convenient to prove the following result which includes Theorem 1.

THEOREM 2. Suppose that $B(x)$ is an operator valued function that is analytic on the real axis. Suppose further that $B(x)$ is selfadjoint for all real $x$. Then the following are equivalent.

(i) $\left[B^{\prime}(x), B(x)\right]=0$ for all real $x$.

(ii) $[B(x), B(y)]=0$ for all real $x$ and $y$.

Proof. Clearly (ii) implies (i). Assume then that (i) holds. Now $B(x)$ admits a series expansion $\sum_{m=0}^{\infty} B_{m} x^{m}$ near zero. Repeated differentiations with respect to $x$ show $B_{m}$ is selfadjoint for every $m$. Following Hellman [5] we insert the series for $B(x)$ into $\left[B^{\prime}(x), B(x)\right]=0$ and get

(1) $\left[B_{1}, B_{0}\right]=0$,

(2) $2\left[B_{2}, B_{0}\right]=0$,

(3) $3\left[B_{3}, B_{0}\right]+\left[B_{2}, B_{1}\right]=0$,

(4) $4\left[B_{4}, B_{0}\right]+2\left[B_{3}, B_{1}\right]=0$,

(5) $5\left[B_{5}, B_{0}\right]+3\left[B_{4}, B_{1}\right]+\left[B_{3}, B_{2}\right]=0, \cdots$.

We wish to show that $\left[B_{i}, B_{j}\right]=0$ for all $i, j \geqq 0$. We will say $(\mathrm{P})$ holds for $B_{i}$ if $\left[B_{i}, B_{j}\right]=0$ for all $j$. Now $\left[B_{n}, B_{0}\right]$ is a sum of $\left[B_{i}, B_{j}\right]$ with $0 \leqq i$, $j \leqq n-1$. Thus if $\left[B_{0}, B_{k}\right]=0$ for $k<n$, then $\left[B_{0},\left[B_{0}, B_{n}\right]\right]=0$ and hence $\left[B_{0}, B_{n}\right]=0$ by Lemma 1 . But $\left[B_{1}, B_{0}\right]=\left[B_{2}, B_{0}\right]=0$. Thus $(\mathrm{P})$ holds for $B_{0}$ by induction. But then $\left[B_{2}, B_{1}\right]=\left[B_{3}, B_{1}\right]=0$ by (3) and (4). Furthermore $\left[B_{n}, B_{1}\right]$ is a linear combination of $\left[B_{i}, B_{j}\right]$ with $1 \leqq i, j \leqq n-1$. As before we get $(\mathrm{P})$ holds for $B_{1}$. Continuing in this manner we get that $(\mathrm{P})$ holds for all $B_{i}$. Thus (ii) holds for all $x$ by analytic continuation.

To see that Theorem 2 implies Theorem 1, let $B(x)=\int_{0}^{x} A(s) d s$ where $A(s)$ is selfadjoint. By Theorem $2,\left[\int_{0}^{x} A(s) d s, \int_{0}^{y} A(s) d s\right]=0$ for all $x$ and $y$ if and only if $\left[\int_{0}^{x} A(s) d s, A(x)\right]=0$ for all $x$. But $\left[\int_{0}^{x} A(s) d s\right.$, $\left.\int_{0}^{y} A(s) d s\right]=0$ for all $x$ and $y$ if and only if $[A(x), A(y)]=0$ for all $x$ and $y$. The if part of this last statement is clear. To get the only if, differentiate $\left[\int_{0}^{x} A(s) d s, \int_{0}^{y} A(s) d y\right]=0$ first with respect to $x$ and then with respect to $y$.

2. Necessity of assumptions. The assumption that $A(x)$ is selfadjoint is necessary. The following example is a modification of one of Hellman's. 
EXAMPLE 1. Let

$$
A(x)=\left(\begin{array}{cccc}
0 & 0 & 0 & 0 \\
0 & 0 & 1 & 2 x \\
4 x^{3} & 0 & 0 & 0 \\
9 x^{2} & 0 & 0 & 0
\end{array}\right)
$$

Then a straightforward calculation verifies $\left[A(x), \int_{0}^{x} A(y) d y\right]=0$. Now the coefficients of the $x$ and $x^{2}$ terms in the power series expansion of $A$ do not commute. Hence $[A(x), A(y)]$ is not always zero.

3. Applications. We review briefly the notation of [3] and [4]. Let $T$ be an operator such that $\|T\| \leqq 1$ and the spectral radius, $r(T)$, is less than one. If $U_{T}(w)$ is the Potapov inner function for $T,|w| \leqq 1$, and $z=i(1-w) /(1+w)$, then let $U_{T}(z)=U_{T}(w)$. The variable $x$ denotes real values of $z . U_{T}(z)$ satisfies the differential equation $U_{T}^{\prime}(x)=i A_{T}(x) U_{T}(x)$ where $A_{T}(x) \geqq 0$ for all $x$. A discussion of Potapov inner functions may be found in [10]. The differential equation was introduced in [6] and extensively studied in [3]. The connection between inner functions and operators is nicely explained in [8].

Combining the results of [1], [4], and this paper we get the following theorem.

THEOREM 3. Suppose that $\|T\|<1$. Let $U_{T}(z)$ be the Potapov inner function of $T$ on the upper half-plane and $A_{T}(x)$ be defined by $U_{T}^{\prime}(x)=$ $i A_{T}(x) U_{T}(x)$. Then the following are equivalent.

(a) $T$ is normal.

(b) $\left[U_{T}(x), U_{T}(y)\right]=0$ for all real $x$ and $y$.

(c) $\left[A_{T}(x), A_{T}(y)\right]=0$ for all real $x$ and $y$.

(d) $\left[A_{T}(x), \int_{0}^{x} A_{T}(y) d y\right]=0$ for all real $x$.

(e) $U_{T}(x)=\exp \left(i \int_{0}^{x} A_{T}(y) d y\right)$.

(f) $\left[U_{T}(x), U_{T}^{\prime}(x)\right]=0$ for all real $x$.

(g) $\left[A_{T}(x), U_{T}(x)\right]=0$ for all real $x$.

(h) $\left[A_{T}(x), U_{T}^{\prime}(x)\right]=0$ for all real $x$.

Proof. The equivalence of (a) and (b) is due to Sherman [12]. The equivalence of (d), (e), (f), (g), and (h) was shown in [1], while (a) and (c) were shown equivalent in [4]. Theorem 1 gives us that (c) and (d) are equivalent.

4. Discussion. It had beeen initially hoped in [1] that the Potapov inner function for some nonnormal operators would satisfy some type of commutation property and hence be easier to work with. Theorem 3 shows 
that most of the obvious ones are equivalent to the normality of $T$ if $\|T\|<1$. If $\|T\|=1$ and $r(T)<1$, then (c) does not necessarily imply (a), while (a) always implies (c). An example was given in [4]. That example was $T=\left[\begin{array}{ll}0 & 1 \\ 0 & 0\end{array}\right]$.

There remains then the possibility that Theorem 1 of [1] could be useful in studying nonspectraloid operators for which (c) holds. It would be of interest, as the next theorem shows, to characterize those operators for which (c) holds.

THEOREM 4. If $\|T\| \leqq 1, r(T)<1$, and $\left[A_{T}(x), A_{T}(y)\right]=0$ for all $x$ and $y$, then $T$ has an invariant subspace.

Proof. Recall from [1, Theorem 7], that the closure of the range of $A_{T}(x)$ is independent of $x$. If there exists an $x_{0}$ such that $A_{T}\left(x_{0}\right)$ is not a scalar multiple of a projection, then let $P$ be a nontrivial projection in the spectral resolution of $A_{T}\left(x_{0}\right)$. Then $\left[A_{T}(x), P\right]=0$ for all $x$ and $A_{T}(x)=$ $A_{T}(x) P \oplus A_{T}(x)(I-P)=A_{1}(x) \oplus A_{2}(x)$. Let $U_{1}(x)$ and $U_{2}(x)$ be the solutions of $X^{\prime}=i A_{1} X, X(0)=P$, and $X^{\prime}=i A_{2} X, X(0)=(I-P)$, respectively. Then $U_{T}(x)$ and $\left(U_{1} \oplus(I-P)\right)\left(P \oplus U_{2}\right) U_{T}(0)$ both satisfy the initial value problem $X^{\prime}=i A_{T} X, X(0)=U_{T}(0)$, and hence are equal. Thus $U_{T}$ factors and $T$ has an invariant subspace. There remains the possibility that $A_{T}(x)=$ $p(x) P$ for a scalar function $p(x)$ and projection $P$. In this case $U_{T}(x)=$ $(q(x) P \oplus(I-P)) U_{T}(0)$ where $q$ is the scalar inner function satisfying $q^{\prime}(x)=i p(x) q(x), q(0)=1$. Clearly $U_{T}$ factors unless it is trivial, that is, $\operatorname{dim} P h=1$ and $q$ is a single Blaschke factor.

The techniques used in proving Theorem 4 can be adapted to prove the following more general result.

THEOREM 5. Suppose that $\|T\| \leqq 1$ and $r(T)<1$. Let $P$ be the projection onto the closure of the range of $A_{T}(x)$. If there exists an operator $B$ such that $\left[B, A_{T}(x)\right]=0$ for all real $x$ and $B P$ is not a scalar multiple of $P$, then $T$ has an invariant subspace.

The example mentioned earlier shows that one cannot improve Theorem 5 to produce a reducing subspace since $\left[\begin{array}{ll}0 & 1 \\ 0 & 0\end{array}\right]$ has none. If we assume $\|T\|<1$, then Theorem 5 can be modified to prove $T$ has a reducing subspace. See [3, p. 37] for details.

As a first step toward characterizing the nonnormal operators for which (c) holds we show they must be isometric on a nontrivial subspace.

THEOREM 6. SUppose that $1 \geqq\|T\|>r(T)$ and that 1 is not an eigenvalue of $T^{*} T$. Then $\left[A_{T}(x), A_{T}(y)\right]=0$ for all real $x$ and $y$ if and only if $T$ is normal. 
Proof. If $T$ is normal, the result follows from the formula for $A_{T}(x)$ given in $[3, \mathrm{p} .31]$ which expresses $A_{T}(x)$ in terms of $T$ and $T^{*}$. Assume then that $1 \geqq\|T\|>r(T)$ and 1 is not an eigenvalue of $T^{*} T$. The case $\|T\|<1$ was done in [4], so assume $\|T\|=1$. Let $D=\left[I-T^{*} T\right]^{1 / 2}$. Then $D$ is a one-to-one selfadjoint operator with a dense range. Up to a scalar function $A_{T}(x)$ is $D(I-w T)^{-1}\left(w-T^{*}\right)^{-1} D$. Expressing this as a Laurent series convergent for $1>|w|>r(T)$, we get $\sum_{n=-\infty}^{-1} D B T^{*-n-1} D w^{n}+$ $\sum_{n=0}^{\infty} D T^{n+1} B D w^{n}$ where $B=\sum_{m=0}^{\infty} T^{m} T^{* m}$. By analytic continuation $\left[A_{T}(z), A_{T}(u)\right]=0$ for $z, u$ such that $1>|(z-i) /(z+i)|,|(u-i) /(u+i)|>$ $r(T)$. Hence all the coefficients in the Laurent series commute. In particular, $D T^{i} B D^{2} B D=D B D^{2} T^{i} B D$. Hence

Now

$$
T^{i} B D^{2}=B D^{2} T^{i} \quad \text { or } \quad\left[T^{i}, B D^{2}\right]=0 .
$$

Hence

and thus

$$
D T^{i} B D^{2} B T^{* j} D=D B T^{* j} D^{2} T^{i} B D
$$

$$
T^{i} B D^{2} B T^{* j}=B T^{* j} D^{2} T^{i} B
$$

$$
D^{2} T^{i} B T^{* j}=T^{* j} D^{2} T^{i} B \text { or }\left[T^{* j}, D^{2} T^{i} B\right]=0 .
$$

Setting $i=j=1$ gives $D^{2} T B T^{*}=T^{*} D^{2} T B$. Multiply both sides by $T^{*}$ on the right and use the identity $T B T^{*}=B-I$ to get $D^{2}(B-I) T^{*}=$ $T^{*} D^{2}(B-I)$. But $D^{2} B T^{*}=T^{*} D^{2} B$ and hence $\left[T^{*}, D^{2}\right]=0$. Thus $T$ and $T^{*}$ commute with $T^{*} T$, which in turn implies that $T$ and $T^{*}$ commute with $B$ also. The earlier identity $D^{2} T B T^{*}=T^{*} D^{2} T B$ now gives $T T^{*}=T^{*} T$, that is, that $T$ is normal.

Theorem 6 improves Theorem 6 of [4] and is probably about the best possible for inner functions analytic on the closed disc.

The assumption that 1 is not an eigenvalue of $T^{*} T$ is equivalent to assuming that $\|T \phi\|<\|\phi\|$ for all nonzero $\phi$ in $h$.

\section{REFERENCES}

1. Stephen L. Campbell, The exponential representation of operator valued, differentiable inner functions, J. Differential Equations 12 (1972), 455-461.

2. - Inner functions analytic at a point, Illinois J. Math. 16 (1972), 651-652.

3. - Operator valued inner functions, Dissertation, Northwestern University, Evanston, Ill., 1972.

4. - Operator valued inner functions analytic on the closed disc, Pacific J. Math. 40 (1972), 57-62.

5. Morton J. Hellman, Matrix commutator relationships arising from systems of linear ordinary differential equations, Dissertation, New York University, New York, 1954. 
6. Henry Helson, The differential equation of an inner function, Studia Math. 35 (1970), 311-321. MR 45 \#5797.

7. - The differential equation of an inner function. II (preprint).

8. - Lectures on invariant subspaces, Academic Press, New York, 1964. MR 30 \#1409.

9. Domingo Antonio Herrero, A geometric approach to inner function-operators and their differential equations (preprint).

10. S. Lynn Jackson, Operators and inner functions, Dissertation, University of California, Berkeley, Calif., 1967.

11. C. R. Putnam, Commutation properties of Hilbert space operators and related topics, Ergebnisse der Math. und ihrer Grenzgebiete, Band 36, Springer-Verlag, New York, 1967. MR 36 \#707.

12. Malcolm J. Sherman, A spectral theory for inner functions, Trans. Amer. Math. Soc. 135 (1969), 387-398. MR 38 \#5037.

Department of Mathematics, North Carolina State University, Raleigh, NORTH CAROLINa 27607 